\title{
Spontaneous thrombosis of cerebral arteriovenous malformation post COVID-19
}

\author{
Sofia Grenho Rodrigues ${ }^{1}\left[\right.$ [ $\cdot$ Renato Oliveira $^{1} \cdot$ Pedro Vilela $^{2} \cdot$ Sofia Nunes Oliveira ${ }^{1}$
}

Received: 4 May 2021 / Accepted: 6 September 2021 / Published online: 15 September 2021

(c) Fondazione Società Italiana di Neurologia 2021

\section{Introduction}

COVID-19 is associated with endothelial dysfunction leading to prothrombotic phenomenon and systemic inflammation [1] and has been associated with intracranial arterial stroke and venous thrombosis.

Brain arteriovenous malformations (AVM) are vascular malformations composed of abnormal arteriovenous shunts at the AVM nidus, mainly fed by pial arteries that do not supply the brain tissue and draining into brain veins. The major clinical presentations are hemorrhage, followed by seizures, neurological deficits, and headaches [2].

Spontaneous resolution of AVM is a rare phenomenon and might result from feeding arteries or drainer veins occlusion, with many pathologic mechanisms proposed, including prothrombotic and hypercoagulable states.

We present a case of spontaneous thrombosis of a cerebral AVM, due to progressive venous drainage thrombosis, in a COVID-19 patient.

\section{Case report}

A 70-year-old man presented to emergency department after sudden onset of left-sided body weakness with associated focal seizures, evolving for $1 \mathrm{~h}$.

This episode occurred 16 days after COVID-19 diagnosis. He was hospitalized for 9 days with SARS-CoV-2 pneumonia and was started on dexamethasone $6 \mathrm{mg}$. He required supplementary oxygen for 7 days. At this hospitalization, his blood routines revealed elevated D-dimer level of $1.69 \mathrm{mg} /$

Sofia Grenho Rodrigues

anasofia.grodrigues@gmail.com

1 Department of Neurology, Hospital da Luz de Lisboa, Lisbon, Portugal

2 Department of Neuroradiology, Hospital da Luz de Lisboa, Lisbon, Portugal $\mathrm{dl}$ (reference level $<0.50 \mathrm{mg} / \mathrm{dl}$ ). His personal history was also relevant for dyslipidemia, obesity, and active smoking. He had no previous neurologic or neurovascular disease.

On admission he presented left homonymous hemianopia and left hemiparesis with Medical Research Council (MRC) grade $3 / 5$. He had clonic movements of the left arm. His laboratory routines were normal, and coagulation studies revealed slightly elevated D-dimer levels $(0.69 \mathrm{mg} / \mathrm{dl})$. There were no further analytic abnormalities, including remain coagulation studies, infectious serologies, genetic thrombophilias, and auto-immune conditions. There were no clinical or laboratory signs of dehydration.

Head CT with CT angiography and brain MRI (Figs. 1 and 2) disclosed a thrombosed right parietal pial arteriovenous malformation, feed by the right anterior cerebral artery and by the inferior division of the medium cerebral artery, with signs of venous drainage thrombosis and perinidal edema. There was an additional small perinidal hematoma (10-mm diameter). Digital subtraction angiography confirmed the progressive thrombosis of the venous drainage of the brain AVM, with no early arteriovenous shunt, and with significant flow stagnation at the arterial feeders and without flow within AVM nidus (Fig. 3).

He was hospitalized and started on levetiracetam $1000 \mathrm{mg}$, twice daily. During hospitalization he presented fluctuation of consciousness and EEG revealed epileptic activity. Levetiracetam was increased to $1500 \mathrm{mg}$, twice daily, and lacosamide $100 \mathrm{mg}$ twice daily was initiated, with clinical and electrographic seizure remission.

A follow-up brain MRI at day 5 of admission revealed increased perilesional edema, and the patient was started on dexamethasone. During the remain hospitalization, he presented a favorable evolution with improvement of the hemiparesis (MRC 4/5) and without further signs of epileptic activity. He was discharged 12 days later. 


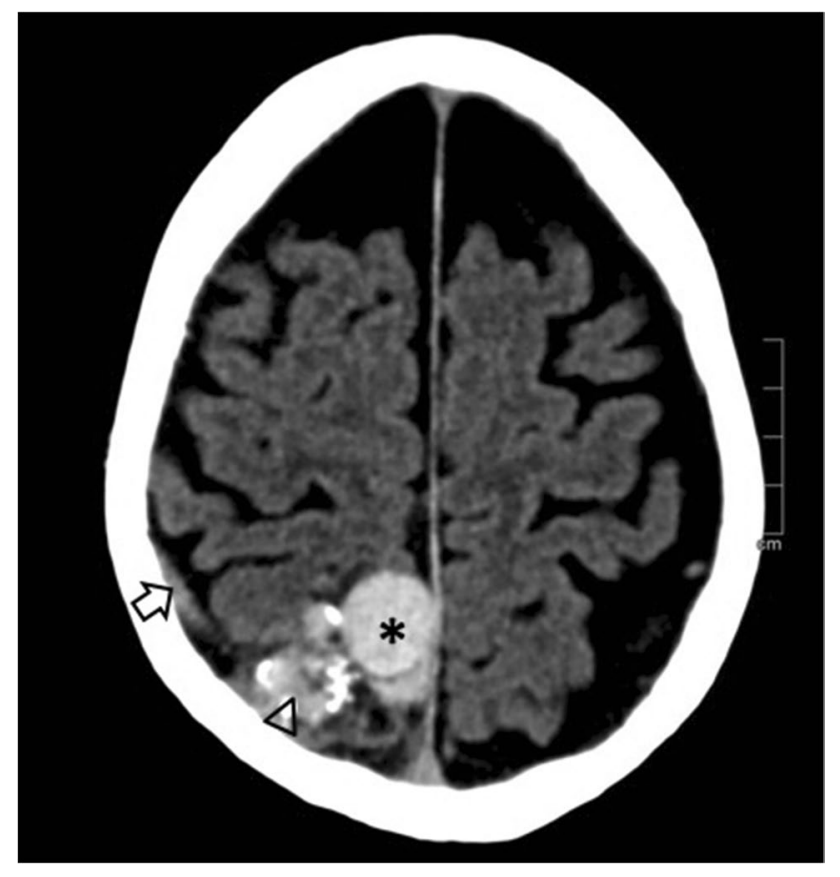

Fig. 1 Non-contrast head CT axial image, showing the spontaneous hyperattenuation of a thrombosed enlarged medial parietal draining vein $($ star $*)$, a partially thrombosed lateral parieto-temporal cortical vein (open arrow), and the nidus (arrowhead) of the brain AVM

\section{Discussion}

We report a clinical case of a spontaneous brain AVM thrombosis due to venous drainage thrombosis, occurring 2 weeks after SARS-CoV-2 pneumonia.
Multiple reports highlight the hypercoagulable state underlying COVID-19, coming from multifactorial pathogeny: immobilization, endothelial cell dysfunction, and platelet activation and inflammatory status. Endothelial dysfunction arises from SARS-CoV-2 vascular attack via angiotensin-converting enzyme 2 receptor and leads to platelet aggregation and thrombosis. Additionally, there is a recruitment of inflammatory cells to the damaged endothelium and to endodermis of vascular wall, with inflammatory infiltration [3]. Patients frequently present elevated coagulation markers such as D-dimer levels, and even slight increases in this marker seem to predict a worst clinical outcome [4] and might predispose to the development of intracranial venous thrombosis.

Spontaneous occlusion of AVM is a rare phenomenon, occurring in $<1 \%$ of all AVM [5], and can be the consequence of two different mechanisms: arterial or vein occlusion. Arterial occlusion result from AVM hemorrhage and associated edema mass effect, thromboembolic events, or atherosclerotic occlusions. Occlusion of AVM draining veins can also result from hematoma mass effect, can be occluded by a clot as a result of hypercoagulative state, or can result from venous stenosis with consequent restriction to venous drainage. According to these, some authors hypothesize that occlusion of AVM draining veins most frequently result in permanent obliteration with increased risk of hemorrhage [5].

Previous studies highlighted common features of the thrombosed AVM that included small nidus defined as smaller than $60 \mathrm{~mm}$, single draining vein, or single arterial feeders and hypercoagulable states [5].

The authors speculate that this case represents a prothrombotic complication of COVID-19, which led to
Fig. 2 Brain MRI axial (A) and coronal (B) T1 WI images, demonstrating the absence of the nidus and venous flow void, confirming the presence of acute thrombosis (depicted as an intraluminal iso-hyper signal) of the brain AVM nidus venous drainage

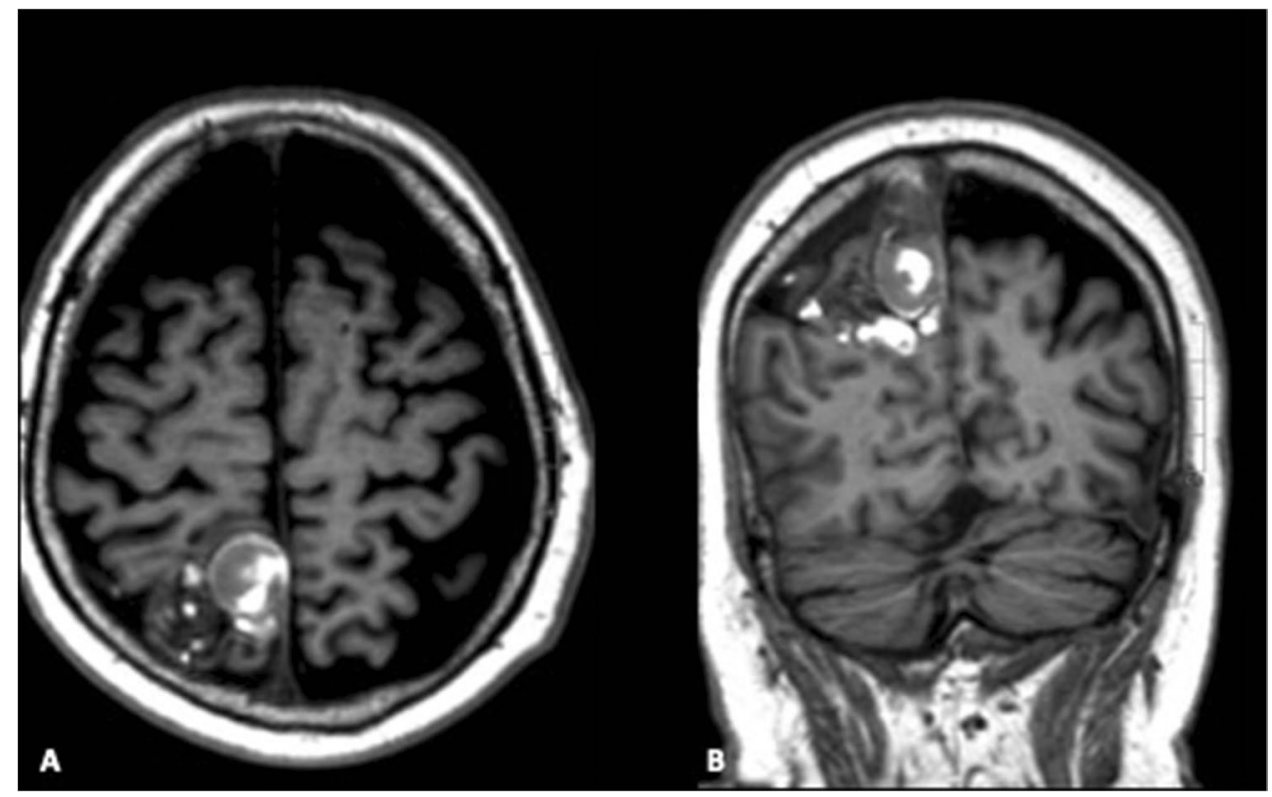


Fig. 3 A and B Lateral view of the right internal carotid artery digital subtraction angiogram (DSA). C and D Lateral view of the left internal carotid DSA. A Late arterial phase showing the absence of AV shunt and early draining veins. B Venous phase showing the absence of filling of the medial parietal vein and the partial thrombosis of the parieto-temporal lateral cortical vein (arrowhead). There was a fetal pattern with posterior cerebral artery filling mainly through the internal carotid artery. C Late arterial phase and venous phase. D Contrast stagnation inside the anterior cerebral artery feeders and nidus (arrowhead), without the presence of patent AV shunts and without early draining veins

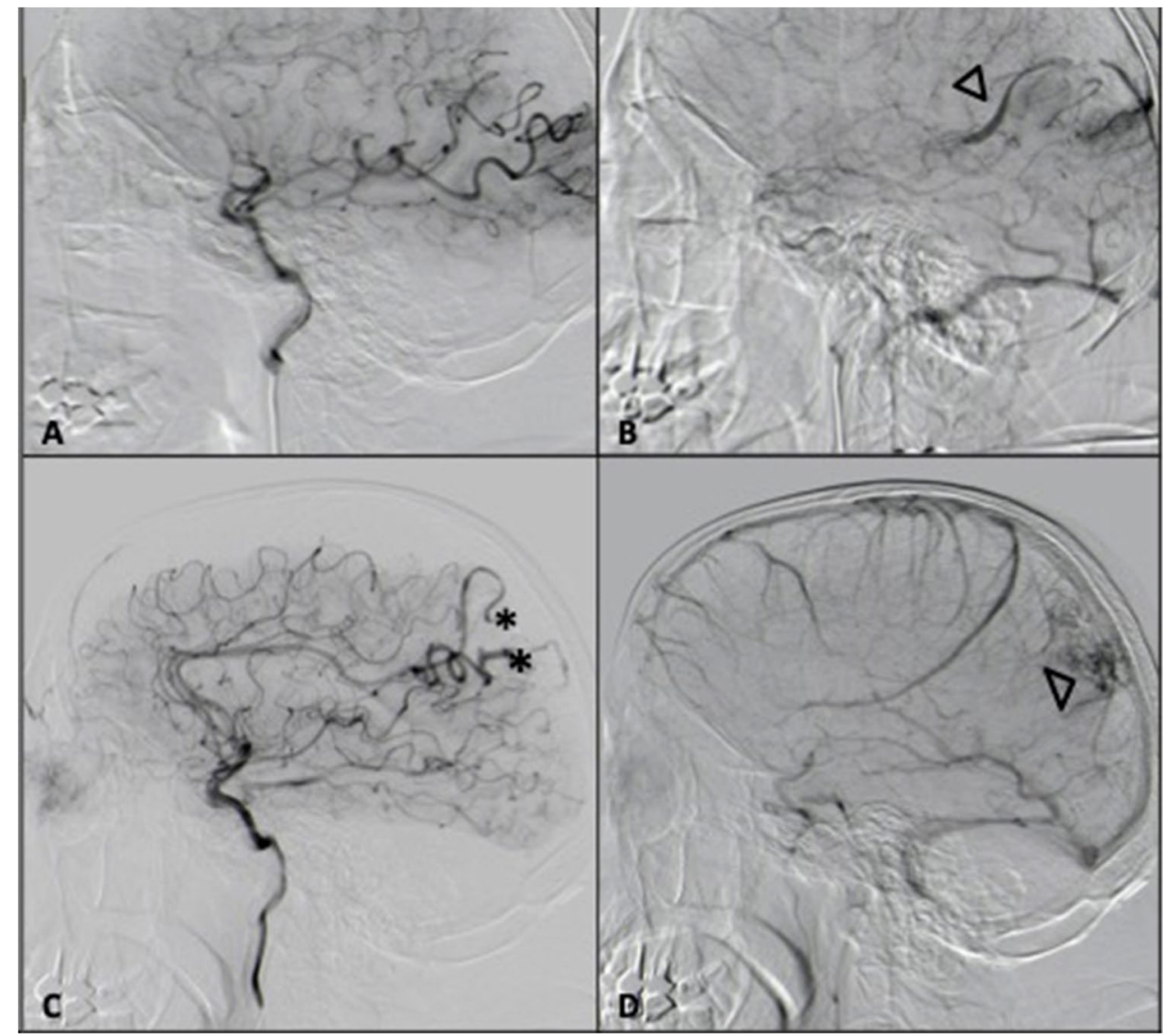

thrombosis of the AVM draining veins and subsequent AVM hemorrhage. This cascade of events resulted ultimately in patient's symptomology-focal signs and de novo epilepsy. The particularity is the favorable outcome due to spontaneous thrombosis and cure of a brain AVM. To our knowledge, this is the first case of spontaneous thrombosis of cerebral AVM in the setting of SARS-CoV-2 infection.

\section{Declarations}

Conflict of interest The authors declare that they have no conflict of interest.

\section{References}

1. Chang R, Mamun A, Dominic A, Le N-T (2021) SARS-CoV-2 Mediated endothelial dysfunction: the potential role of chronic oxidative stress. Front Physiol 1-27. https://www.frontiersin.org/ articles/10.3389/fphys.2020.605908/full
2. Chen C-J, Ding D, Derdeyn CP, et al (2020) Brain arteriovenous malformations. Neurology 95(20):917-927. http://www.neuro logy.org/lookup/doi/10.1212/WNL.0000000000010968

3. Janardhan V, Janardhan V, Kalousek V (2020) COVID-19 as a blood clotting disorder masquerading as a respiratory illness: a cerebrovascular perspective and therapeutic implications for stroke thrombectomy. J Neuroimaging 30(5):555-561. https:// onlinelibrary.wiley.com/doi/10.1111/jon. 12770

4. Tang N, Li D, Wang X, Sun Z (2020) Abnormal coagulation parameters are associated with poor prognosis in patients with novel coronavirus pneumonia. J Thromb Haemost 18(4):844-847. https://onlinelibrary.wiley.com/doi/abs/10.1111/jth.14768

5. Lee S, Vilela P, Willinsky R, TerBrugge K (2002) Spontaneous regression of cerebral arteriovenous malformations: clinical and angiographic analysis with review of the literature. Neuroradiology 44(1):11-16. http://link.springer.com/10.1007/s002340100 702

Publisher's note Springer Nature remains neutral with regard to jurisdictional claims in published maps and institutional affiliations. 